\title{
Singular boundary value problems with integrable singularities
}

\author{
R. P. Agarwal and Donal O'Regan
}




\title{
SINGULAR BOUNDARY VALUE PROBLEMS WITH INTEGRABLE SINGULARITIES
}

\author{
R. P. AGARWAL AND DONAL O'REGAN
}

[Received: December 6, 2003]

\begin{abstract}
Aвstract. A new technique based on Schauder's fixed point theorem is given which establishes new existence results for singular two point boundary value problems where the singularity is integrable.
\end{abstract}

Mathematics Subject Classification: 34B15, 34B16, 34B18

Keywords: Singular problem, two-point problem, integrable singularity, Schauder theorem

\section{InTRODUCTION}

This paper presents a new approach to establishing the existence for the Dirichlet boundary value problem

$$
\begin{aligned}
y^{\prime \prime}+f\left(t, y, y^{\prime}\right) & =0 \quad \text { a. e. on }[0,1] \\
y(0)=y(1) & =0
\end{aligned}
$$

where our nonlinearity $f$ may be singular in the independent variable and may also be singular at $y=0$. Problems of the form (1.1) have received a lot of attention in the literature; see [1-4] and the references therein. This paper presents a new approach based on Schauder's fixed point theorem and our results extend and complement those in the literature. Moreover it is easy to see that we could consider SturmLiouville boundary data in (1.1); however, since the arguments are essentially the same we will restrict our discussion to Dirichlet data. In Section 2, we also discuss a more general situation, namely

$$
\begin{aligned}
y^{\prime \prime}+f\left(t, y, y^{\prime}\right) & =0 \quad \text { a. e. on }[0,1] \\
y(0)=y^{\prime}(1) & =0
\end{aligned}
$$

where our nonlinearity $f$ may be singular in the independent variable and may also be singular at $y=0$ and $y^{\prime}=0$. Only a handful of papers (see $[\mathbf{1 , 4}]$ ) discuss the situation when $f$ may be singular at $y=0$ and $y^{\prime}=0$. 


\section{EXISTENCE THEORY}

Our first results concern

$$
\begin{aligned}
y^{\prime \prime}+f\left(t, y, y^{\prime}\right) & =0 \quad \text { a. e. on }[0,1] \\
y(0)=y(1) & =0,
\end{aligned}
$$

where $f(t, y, z)$ may be singular at $y=0$. We note that $f$ may be singular also in the independent variable at some set $\Omega \subseteq[0,1]$ with measure zero.

Theorem 2.1. Suppose the following conditions are satisfied:

(2.2) $f:[0,1] \times(0, \infty) \times \mathbb{R} \rightarrow \mathbb{R}$ with $t \mapsto f(t, y, z)$ measurable for every $(y, z) \in$ $(0, \infty) \times \mathbb{R}$ and $(y, z) \mapsto f(t, y, z)$ continuous for a. e. $t \in(0,1)$;

(2.3) for any $r>0, \exists \psi_{r}:[0,1] \rightarrow \mathbb{R}, \psi_{r}>0$ a. e. on $[0,1], \psi_{r} \in L^{1}[0,1]$ with $f(t, y, z) \geq \psi_{r}(t)$ a. e. on $[0,1]$ for every $y \in(0, r]$ and $z \in[-r, r]$;

(2.4) for any $r>0$ with $\int_{0}^{1} G(t, s) \psi_{r}(s) d s \leq r$ for $t \in[0,1], \exists h_{r}:[0,1] \rightarrow \mathbb{R}$, $h_{r} \geq 0$ a. e. on $[0,1], h_{r} \in L^{1}[0,1]$ with $f(t, y, z) \leq h_{r}(t)$ for a. $e . t \in[0,1]$ and $y \in\left[\int_{0}^{1} G(t, s) \psi_{r}(s) d s, r\right]$ and $z \in[-r, r]$, where

$$
G(t, s)= \begin{cases}(1-t) s, & 0 \leq s \leq t \leq 1 \\ (1-s) t, & 0 \leq t \leq s \leq 1\end{cases}
$$

(2.5) $\exists M>0$ with $M \geq \int_{0}^{1} h_{M}(s) d s$ and $h_{M}(s) \geq \psi_{M}(s)$ for a. e. $s \in[0,1]$.

Then (2.1) has a solution $y \in W^{2,1}[0,1]$ with $y(t)>0$ for $t \in(0,1)$.

Remark 2.1. In Theorem 2.1, it is possible to replace (2.4) by the following condition:

(2.6) for any $r>0$ with $\int_{0}^{1} G(t, s) \psi_{r}(s) d s \leq r$ for $t \in[0,1]$, assume that $h_{r} \in$ $L^{1}[0,1]$ where

$$
h_{r}(t)=\sup \left\{f(t, y, z): y \in\left[\int_{0}^{1} G(t, s) \psi_{r}(s) d s, r\right] \text { and } z \in[-r, r]\right\} \text {. }
$$

Proof. Choose $M$ so that (2.3), (2.4), and (2.5) hold. Let

$$
\begin{gathered}
Q=\left\{u \in C^{1}[0,1]: \int_{0}^{1} G(t, s) \psi_{M}(s) d s \leq u(t) \leq \int_{0}^{1} G(t, s) h_{M}(s) d s\right. \\
\text { and } \left.\left|u^{\prime}(t)\right| \leq M \text { for } t \in[0,1]\right\} .
\end{gathered}
$$

Clearly $Q$ is a closed, convex subset of $C^{1}[0,1]$. To establish our result, we will apply Schauder's fixed point theorem to the operator $T: C^{1}[0,1] \rightarrow C^{1}[0,1]$; here, $T$ is given by the equality

$$
(T y)(t)=\int_{0}^{1} G(t, s) f\left(s, y(s), y^{\prime}(s)\right) d s
$$


We also note that (2.5) guarantees that

$$
M \geq \int_{0}^{1} G(s, s) h_{M}(s) d s .
$$

First we show $T: Q \rightarrow Q$. To see this, let $u \in Q$, so, for $t \in[0,1]$, we have (by (2.5))

$$
\int_{0}^{1} G(t, s) \psi_{M}(s) d s \leq u(t) \leq \int_{0}^{1} G(t, s) h_{M}(s) d s \leq \int_{0}^{1} G(s, s) h_{M}(s) d s \leq M
$$

and $\left|u^{\prime}(t)\right| \leq M$. As a result, (2.3) yields

$$
f\left(s, u(s), u^{\prime}(s)\right) \geq \psi_{M}(s) \text { a. e. on }[0,1],
$$

so

$$
(T u)(t)=\int_{0}^{1} G(t, s) f\left(s, u(s), u^{\prime}(s)\right) d s \geq \int_{0}^{1} G(t, s) \psi_{M}(s) d s \text { for } t \in[0,1] .
$$

Furthermore, (2.4) implies

$$
f\left(s, u(s), u^{\prime}(s)\right) \leq h_{M}(s) \text { a. e. on }[0,1],
$$

so

and (by (2.5))

$$
(T u)(t) \leq \int_{0}^{1} G(t, s) h_{M}(s) d s
$$

$$
\begin{aligned}
\left|(T u)^{\prime}(t)\right| & =\left|-\int_{0}^{t} s f\left(s, u(s), u^{\prime}(s)\right) d s+\int_{t}^{1}(1-s) f\left(s, u(s), u^{\prime}(s)\right) d s\right| \\
& \leq \int_{0}^{t} s h_{M}(s) d s+\int_{t}^{1}(1-s) h_{M}(s) d s \leq \int_{0}^{1} h_{M}(s) d s \leq M
\end{aligned}
$$

for $t \in[0,1]$. Thus, $T: Q \rightarrow Q$. Next we show that $T$ is continuous. Let $y_{n} \in Q$ and $y_{n} \rightarrow y$ in $C^{1}[0,1]$. Then, for $t \in[0,1]$, we have

$$
\begin{aligned}
\left|\left(T y_{n}\right)(t)-(T y)(t)\right| & \leq \int_{0}^{1} G(s, s)\left|f\left(s, y_{n}(s), y_{n}^{\prime}(s)\right)-f\left(s, y(s), y^{\prime}(s)\right)\right| d s \\
& \leq \int_{0}^{1}\left|f\left(s, y_{n}(s), y_{n}^{\prime}(s)\right)-f\left(s, y(s), y^{\prime}(s)\right)\right| d s
\end{aligned}
$$

and

$$
\begin{aligned}
\left|\left(T y_{n}\right)^{\prime}(t)-(T y)^{\prime}(t)\right| & \leq \int_{0}^{t} s\left|f\left(s, y_{n}(s), y_{n}^{\prime}(s)\right)-f\left(s, y(s), y^{\prime}(s)\right)\right| d s \\
& +\int_{t}^{1}(1-s)\left|f\left(s, y_{n}(s), y_{n}^{\prime}(s)\right)-f\left(s, y(s), y^{\prime}(s)\right)\right| d s \\
& \leq \int_{0}^{1}\left|f\left(s, y_{n}(s), y_{n}^{\prime}(s)\right)-f\left(s, y(s), y^{\prime}(s)\right)\right| d s .
\end{aligned}
$$


Thus, the Lebesgue dominated convergence theorem implies

$$
\sup _{t \in[0,1]}\left|\left(T y_{n}\right)(t)-(T y)(t)\right| \leq \int_{0}^{1}\left|f\left(s, y_{n}(s), y_{n}^{\prime}(s)\right)-f\left(s, y(s), y^{\prime}(s)\right)\right| d s \rightarrow 0
$$

as $n \rightarrow \infty$ and

$$
\sup _{t \in[0,1]}\left|\left(T y_{n}\right)^{\prime}(t)-(T y)^{\prime}(t)\right| \leq \int_{0}^{1}\left|f\left(s, y_{n}(s), y_{n}^{\prime}(s)\right)-f\left(s, y(s), y^{\prime}(s)\right)\right| d s \rightarrow 0
$$

as $n \rightarrow \infty$ because

$$
\int_{0}^{1}\left|f\left(s, y_{n}(s), y_{n}^{\prime}(s)\right)-f\left(s, y(s), y^{\prime}(s)\right)\right| d s \leq 2 \int_{0}^{1} h_{M}(s) d s .
$$

As a result, $T: Q \rightarrow Q$ is continuous. It remains to show that $T: Q \rightarrow Q$ is compact. This follows from the Arzela-Ascoli theorem and the following relations (here, $y \in Q$ and $t, t^{\prime} \in[0,1]$ with $t<t^{\prime}$ ):

$$
\begin{gathered}
\sup _{t \in[0,1]}|(T y)(t)| \leq \sup _{t \in[0,1]} \int_{0}^{1} G(s, s) h_{M}(s) \leq M, \\
\sup _{t \in[0,1]}\left|(T y)^{\prime}(t)\right| \leq \sup _{t \in[0,1]} \int_{0}^{1} h_{M}(s) \leq M, \\
\left|(T y)(t)-(T y)\left(t^{\prime}\right)\right| \leq \int_{0}^{1}\left|G(t, s)-G\left(t^{\prime}, s\right)\right| h_{M}(s) d s,
\end{gathered}
$$

and

$$
\left|(T y)^{\prime}(t)-(T y)^{\prime}\left(t^{\prime}\right)\right| \leq \int_{t}^{t^{\prime}} s h_{M}(s) d s+\int_{t}^{t^{\prime}}(1-s) h_{M}(s) d s=\int_{t}^{t^{\prime}} h_{M}(s) d s .
$$

Now Schauder's fixed point theorem guarantees that the mapping $T$ has a fixed point in $Q$.

Our next result is a more "applicable" version of Theorem 2.1.

Theorem 2.2. Suppose that (2.2) and (2.3) hold and, in addition, the following conditions are satisfied:

(2.7) $f(t, y, z) \leq q(t)[g(y)+\tau(y)] \phi(z)$ on $[0,1] \times(0, \infty) \times \mathbb{R}$ with $g>0$ continuous and nonincreasing on $(0, \infty), \tau \geq 0$ continuous and nondecreasing on $(0, \infty)$, $\phi \geq 0$ continuous on $(-\infty, \infty)$, and $q:[0,1] \rightarrow \mathbb{R}$ with $q>0$ a. e. on $[0,1]$;

(2.8) the relation

$$
\int_{0}^{1} q(s) g\left(c_{0} s(1-s)\right) d s<\infty
$$

holds for any $c_{0}>0$; 
(2.9) there exists $M>0$ such that

$$
\begin{aligned}
& \quad M \geq\left[\sup _{w \in[-M, M]} \phi(w)\right] \int_{0}^{1} q(s)\left[\tau(M)+g\left(\int_{0}^{1} G(s, x) \psi_{M}(x) d x\right)\right] d s \\
& \text { and } \\
& \qquad q(t)\left[\tau(M)+g\left(\int_{0}^{1} G(t, s) \psi_{M}(s) d s\right)\right] \sup _{w \in[-M, M]} \phi(w) \geq \psi_{M}(t)
\end{aligned}
$$

for a. e. $t \in[0,1]$.

Then (2.1) has a solution $y \in W^{2,1}[0,1]$ with $y(t)>0$ for $t \in(0,1)$.

Proof. The result follows from Theorem 2.1 once we show (2.4) and (2.5) hold. Notice for a. e. $t \in[0,1]$ and $y \in\left[\int_{0}^{1} G(t, s) \psi_{r}(s) d s, r\right]$ and $z \in[-r, r]$, then (2.7) yields

$$
f(t, y, z) \leq q(t)\left[\tau(r)+g\left(\int_{0}^{1} G(t, s) \psi_{r}(s) d s\right)\right] \sup _{w \in[-r, r]} \phi(w) .
$$

If we take

$$
h_{r}(t)=q(t)\left[\tau(r)+g\left(\int_{0}^{1} G(t, s) \psi_{r}(s) d s\right)\right] \sup _{w \in[-r, r]} \phi(w),
$$

then (2.4) is immediate if we show $h_{r} \in L^{1}[0,1]$. Also (2.9) guarantees (2.5). It remains to show that $h_{r} \in L^{1}[0,1]$. To see this, it is enough to establish the inclusion

$$
q(t) g\left(\int_{0}^{1} G(t, s) \psi_{r}(s) d s\right) \in L^{1}[0,1] .
$$

To show this, notice

$$
\int_{0}^{1} G(t, s) \psi_{r}(s) d s=t(1-t) \Theta_{r}(t)
$$

where

Now since

$$
\Theta_{r}(t)=\frac{1}{1-t} \int_{t}^{1}(1-s) \psi_{r}(s) d s+\frac{1}{t} \int_{0}^{t} s \psi_{r}(s) d s
$$

$$
\left|\frac{1}{t} \int_{0}^{t} s \psi_{r}(s) d s\right| \leq \int_{0}^{t} \psi_{r}(s) d s \rightarrow 0 \text { as } t \rightarrow 0^{+}
$$

and

$$
\left|\frac{1}{1-t} \int_{t}^{1}(1-s) \psi_{r}(s) d s\right| \leq \int_{t}^{1} \psi_{r}(s) d s \rightarrow 0 \text { as } t \rightarrow 1^{-}
$$

we have that $\Theta_{r}$ extends to a continuous function on $[0,1]$. Thus, there exists $k_{r}>0$ with $\Theta_{r}(t) \geq k_{r}>0$ for $t \in[0,1]$. As a result,

$$
\int_{0}^{1} G(t, s) \psi_{r}(s) d s \geq k_{r} t(1-t) \text { for } t \in[0,1]
$$


So

$$
h_{r}(t) \leq q(t)\left[\tau(r)+g\left(k_{r} t(1-t)\right)\right] \sup _{w \in[-M, M]} \phi(w),
$$

and $h_{r} \in L^{1}[0,1]$ from $(2.8)$.

To show how Theorem 2.2 can be applied in practice, consider the problem

$$
\begin{aligned}
y^{\prime \prime}+q(t)[g(y)+\tau(y)] \phi\left(y^{\prime}\right) & =0 \quad \text { a. e. on }[0,1], \\
y(0)=y(1) & =0 .
\end{aligned}
$$

Theorem 2.3. Assume that the following conditions are satisfied:

(2.11) $g>0$ is continuous and nonincreasing on $(0, \infty), \tau \geq 0$ is continuous and nondecreasing on $(0, \infty), \phi \geq 0$ is continuous on $(-\infty, \infty), q:[0,1] \rightarrow \mathbb{R}$ is measurable and $q>0$ a. e. on $[0,1]$;

(2.12) $\exists a_{0}>0$ such that $\phi(z) \geq a_{0}$ for $z \in(-\infty, \infty)$;

(2.13) $\int_{0}^{1} q(s) g\left(c_{0} s(1-s)\right) d s<\infty$ for any $c_{0}>0$;

(2.14) there exists $M>0$ for which

$$
M \geq\left[\sup _{w \in[-M, M]} \phi(w)\right] \int_{0}^{1} q(s)\left[\tau(M)+g\left(a_{0} g(M) \int_{0}^{1} G(s, x) q(x) d x\right)\right] d s
$$

$$
\text { and }
$$

$$
\sup _{w \in[-M, M]} \phi(w)\left[\tau(M)+g\left(a_{0} g(M) \int_{0}^{1} G(t, s) q(s) d s\right)\right] \geq a_{0} g(M)
$$

for a. e. $t \in[0,1]$.

Then (2.10) has a solution $y \in W^{2,1}[0,1]$ with $y(t)>0$ for $t \in(0,1)$.

Proof. The result follows from Theorem 2.2 once we notice that we can take $\psi_{r}(t)=q(t) g(r) a_{0}$

Remark 2.2. It is possible to replace (2.12) in Theorem 2.3 by the assumption

$$
\text { for any } r>0, \exists a_{r}>0 \text { with } \phi(z) \geq a_{r} \text { for } z \in[-r, r]
$$

provided $a_{0}$ in (2.14) is replaced by $a_{M}$.

Remark 2.3. If $g(y)=y^{-\alpha}, \alpha>0$ and for $x \geq 0$ we have $\tau(x)=A x^{p}+B, A \geq 0$, $B \geq 0, p \geq 0$ and for $w \in \mathbb{R}$ we have $\phi(w)=C|w|^{q}+D, C \geq 0, D \geq 0, q \geq 0$ then (2.14) is satisfied if there exists a $M>0$ with

$$
M \geq\left(C M^{q}+D\right) \int_{0}^{1} q(s)\left[A M^{p}+B+M^{\alpha^{2}}\left(a_{0} \int_{0}^{1} G(s, x) q(x) d x\right)^{-\alpha}\right] d s
$$

and

$$
\left[C M^{q}+D\right]\left[A M^{p}+B+M^{\alpha^{2}}\left(a_{0} \int_{0}^{1} G(t, x) q(x) d x\right)^{-\alpha}\right] d s \geq a_{0} M^{-\alpha}
$$


for a. e. $t \in[0,1]$. Of course if $\alpha^{2}+q<1$ and $q+p<1$, then this inequality is satisfied for $M$ large.

As we remarked in the introduction, we could discuss Sturm Liouville data instead of Dirichlet data in (2.1). If the partial derivative of Green's function with respect to the first variable is of fixed sign, we can improve Theorem 2.1 considerably. To show what can be done, we consider the boundary value problem

$$
\begin{aligned}
y^{\prime \prime}+f\left(t, y, y^{\prime}\right) & =0 \quad \text { a. e. on }[0,1], \\
y(0)=y^{\prime}(1) & =0 .
\end{aligned}
$$

It is easy to state and prove an analogue of Theorem 2.1 for (2.15). However, we will prove more since we will assume $f(t, y, z)$ may be singular at $y=0$ and $z=0$.

Theorem 2.4. Suppose that the following conditions are satisfied:

(2.16) $f:[0,1] \times(0, \infty) \times(0, \infty) \rightarrow \mathbb{R}$ with $t \mapsto f(t, y, z)$ measurable for every $(y, z) \in(0, \infty) \times(0, \infty)$ and $(y, z) \mapsto f(t, y, z)$ continuous for a. e. $t \in(0,1)$;

(2.17) for any $r>0, \exists \psi_{r}:[0,1] \rightarrow \mathbb{R}, \psi_{r}>0$ a. e. on $[0,1], \psi_{r} \in L^{1}[0,1]$ with $f(t, y, z) \geq \psi_{r}(t) a$. e. on $[0,1]$ for every $y \in(0, r]$ and $z \in(0, r]$;

(2.18) for any $r>0$ with $\int_{0}^{1} \psi_{r}(s) d s \leq r, \exists h_{r}:[0,1] \rightarrow \mathbb{R}, h_{r} \geq 0$ a.e. on $[0,1], h_{r} \in L^{1}[0,1]$ with $f(t, y, z) \leq h_{r}(t)$ for a. e. $t \in[0,1], y \in$ $\left[\int_{0}^{1} k(t, s) \psi_{r}(s) d s, r\right]$ and $z \in\left[\int_{t}^{1} \psi_{r}(s) d s, r\right]$, where

$$
k(t, s)= \begin{cases}s, & 0 \leq s \leq t \leq 1 \\ t, & 0 \leq t \leq s \leq 1\end{cases}
$$

(2.19) $\exists M>0$ with $M \geq \int_{0}^{1} h_{M}(s) d s$ and $h_{M}(s) \geq \psi_{M}(s)$ for a. $e . s \in[0,1]$;

Then (2.15) has a solution $y \in W^{2,1}[0,1]$ with $y(t)>0$ for $t \in(0,1]\left(\right.$ and $y^{\prime}(t)>0$ for $t \in[0,1))$.

Proof. Choose $M$ so that (2.17), (2.18), and (2.19) hold. Let

$$
\begin{aligned}
(T y)(t) & =\int_{0}^{1} k(t, s) f\left(s, y(s), y^{\prime}(s)\right) d s \\
& =\int_{0}^{t} s f\left(s, y(s), y^{\prime}(s)\right) d s+t \int_{t}^{1} f\left(s, y(s), y^{\prime}(s)\right) d s
\end{aligned}
$$

and

$$
\begin{gathered}
Q=\left\{u \in C^{1}[0,1]: \int_{0}^{1} k(t, s) \psi_{M}(s) d s \leq u(t) \leq \int_{0}^{1} k(t, s) h_{M}(s) d s\right. \\
\text { and } \left.\int_{t}^{1} \psi_{M}(s) d s \leq u^{\prime}(t) \leq \int_{t}^{1} h_{M}(s) d s \text { for } t \in[0,1]\right\} .
\end{gathered}
$$

Notice that (2.19) yields $M \geq \int_{0}^{1} s h_{M}(s) d s$. If $u \in Q$, then (2.17) (and (2.19)) implies

$$
f\left(s, u(s), u^{\prime}(s)\right) \geq \psi_{M}(s) \text { a. e. on }[0,1],
$$


and (2.18) implies

$$
f\left(s, u(s), u^{\prime}(s)\right) \leq h_{M}(s) \text { a. e. on }[0,1] .
$$

As a result, $T u \in Q$ (note that $(T u)^{\prime}(t)=\int_{t}^{1} f\left(s, y(s), y^{\prime}(s)\right) d s$ ), so $T: Q \rightarrow Q$. Essentially the same reasoning as in Theorem 2.1 guarantees that $T: Q \rightarrow Q$ is continuous and compact.

Our next result is a more "applicable" version of Theorem 2.4.

Theorem 2.5. Suppose that (2.16) and (2.17) hold and, in addition, the following conditions are satisfied:

(2.20) the estimate

$$
f(t, y, z) \leq q(t)[g(y)+\tau(y)][\phi(z)+\lambda(z)]
$$

holds on $[0,1] \times(0, \infty) \times(0, \infty)$ with $g>0, \phi>0$ continuous and nonincreasing on $(0, \infty), \tau \geq 0, \lambda \geq 0$ continuous and nondecreasing on $(0, \infty)$, $q:[0,1] \rightarrow \mathbb{R}$ with $q>0$ a. e. on $[0,1]$

(2.21) the inequality

$$
\int_{0}^{1} q(s) g\left(c_{0} s\right) \phi\left(\int_{s}^{1} \psi_{r}(x) d x\right) d s<\infty
$$

is true for any $r>0$ and $c_{0}>0$;

(2.22) there exists $M>0$ such that

$$
\begin{aligned}
& M \geq \int_{0}^{1} q(s)\left[\tau(M)+g\left(\int_{0}^{1} k(s, x) \psi_{M}(x) d x\right)\right]\left[\lambda(M)+\phi\left(\int_{s}^{1} \psi_{M}(x) d x\right)\right] d s \\
& \quad \text { and } \\
& q(t)\left[\tau(M)+g\left(\int_{0}^{1} k(t, s) \psi_{M}(s) d s\right)\right]\left[\lambda(M)+\phi\left(\int_{t}^{1} \psi_{M}(s) d s\right)\right] \geq \psi_{M}(t) \\
& \quad \text { for a. e. } t \in[0,1] .
\end{aligned}
$$

Then (2.15) has a solution $y \in W^{2,1}[0,1]$ with $y(t)>0$ for $t \in(0,1]$ (and, furthermore, $y^{\prime}(t)>0$ for $\left.t \in[0,1)\right)$.

Proof. This follows from Theorem 2.4 once one notices that one can take

$$
h_{r}(t)=q(t)\left[\tau(r)+g\left(\int_{0}^{1} k(t, s) \psi_{r}(s) d s\right)\right]\left[\lambda(r)+\phi\left(\int_{t}^{1} \psi_{r}(s) d s\right)\right]
$$

for any $r>0$. We need only to check that

$$
q(t) g\left(\int_{0}^{1} k(t, s) \psi_{r}(s) d s\right) \phi\left(\int_{t}^{1} \psi_{r}(s) d s\right) \in L^{1}[0,1] .
$$

To see this, notice that

$$
\int_{0}^{1} k(t, s) \psi_{r}(s) d s=t \Psi_{r}(t)
$$


where

$$
\Psi_{r}(t)=\frac{1}{t} \int_{0}^{t} s \psi_{r}(s) d s+\int_{t}^{1} \psi_{r}(s) d s .
$$

It is easy to see (as in Theorem 2.2) that $\Psi_{r}$ extends to a continuous function on $[0,1]$. Thus, there exists $k_{r}>0$ with $\Psi_{r}(t) \geq k_{r}>0$ for $t \in[0,1]$. As a result, we have

$$
\int_{0}^{1} k(t, s) \psi_{r}(s) d s \geq k_{r} t \text { for } t \in[0,1],
$$

so

$$
h_{r}(t) \leq q(t)\left[\tau(r)+g\left(k_{r} t\right)\right]\left[\lambda(r)+\phi\left(\int_{t}^{1} \psi_{r}(s) d s\right)\right],
$$

and $h_{r} \in L^{1}[0,1]$ from $(2.21)$.

Remark 2.4. There is also an analogue of Theorem 2.3 for the boundary value problem

$$
\begin{aligned}
y^{\prime \prime}+q(t)[g(y)+\tau(y)]\left[\phi\left(y^{\prime}\right)+\lambda\left(y^{\prime}\right)\right] & =0 \quad \text { a. e. on }[0,1], \\
y(0)=y^{\prime}(1) & =0 .
\end{aligned}
$$

We leave the details to the reader.

\section{REFERENCES}

[1] Agarwal, R. P., O’Regan, D. and Wong, P. J. Y.: Positive Solutions of Differential, Difference and Integral Equations, Kluwer Acad. Publ., Dordrecht, 1999.

[2] Bobisud, L. E., Calvert, J. E. and Royalty, W. D.: Some existence results for singular boundary value problems, Differential Integral Equations, 6 (1993), 553-571.

[3] Bonannao, G.: Positive solutions to nonlinear singular second order boundary value problems, Ann. Polonici Mathematici, 64 (1996), 237-251.

[4] O'Regan, D.: Positive solutions to singular boundary value problems with at most linear growth, Applicable Analysis, 49 (1993), 171-196.

\section{Authors' Addresses}

\section{R. P. Agarwal:}

Department of Mathematical Science, Florida Institute of Technology, Melbourne, Florida 32901, USA

\section{Donal O'Regan:}

Department of Mathematics, National University of Ireland, Galway, Ireland 